\title{
Theoretical study on the optoelectronic properties of electron-withdrawing substituted diethynylfluorenyl Gold(I) complexes
}

\author{
Yi Liao $^{a b c}$, Guo-Chun Yang ${ }^{c}$, Ji-Kang Feng $^{* a b}$, Li-Li Shi ${ }^{a}$, Shuang-Yang Yang ${ }^{a}$, Li \\ Yang $^{a}$, Ai-Min Ren ${ }^{a}$ \\ ${ }^{\text {a }}$ State Key Laboratory of Theoretical and Computational Chemistry, Institute of \\ Theoretical Chemistry, Jilin University, Changchun 130023, P. R. China; \\ b The College of Chemistry, Jilin University, Changchun 130023, P. R. China. \\ ${ }^{\mathrm{C}}$ Institute of Functional Material Chemistry, Faculty of Chemistry, Northeast Normal \\ University, Changchun 130024, China
}

Corresponding author. Email: JiKangf@yahoo.com; Fax: +86-431-8945942

* Corresponding author. Email: JiKangf@yahoo.com; Fax: +86-431-8945942. 


\section{Supporting information}

Table S1. B3LYP/E60 calculated structures of 1,2 and 3 in their neutral, cationic and anionic states.

\begin{tabular}{cccccccccc}
\hline & \multicolumn{3}{c}{ AuTFT } & \multicolumn{3}{c}{ AuTFOT } & \multicolumn{3}{c}{ AuTFCNT } \\
\hline & neutral & cation & anion & neutral & cation & anion & neutral & cation & anion \\
\hline $\mathrm{r}(1,2)$ & 1.414 & 1.424 & 1.425 & 1.416 & 1.413 & 1.428 & 1.415 & 1.407 & 1.427 \\
$\mathrm{r}(2,3)$ & 1.414 & 1.421 & 1.431 & 1.412 & 1.430 & 1.430 & 1.410 & 1.423 & 1.428 \\
$\mathrm{r}(3,4)$ & 1.392 & 1.388 & 1.377 & 1.398 & 1.388 & 1.384 & 1.396 & 1.387 & 1.381 \\
$\mathrm{r}(4,11)$ & 1.399 & 1.407 & 1.414 & 1.392 & 1.405 & 1.407 & 1.390 & 1.399 & 1.406 \\
$\mathrm{r}(10,11)$ & 1.413 & 1.421 & 1.429 & 1.412 & 1.434 & 1.427 & 1.419 & 1.437 & 1.435 \\
$\mathrm{r}(1,10)$ & 1.385 & 1.380 & 1.374 & 1.382 & 1.386 & 1.371 & 1.392 & 1.404 & 1.380 \\
$\mathrm{r}(9,10)$ & 1.517 & 1.520 & 1.514 & 1.501 & 1.483 & 1.502 & 1.481 & 1.456 & 1.480 \\
$\mathrm{r}(11,12)$ & 1.464 & 1.449 & 1.432 & 1.477 & 1.445 & 1.445 & 1.463 & 1.441 & 1.432 \\
$\mathrm{r}(7,14)$ & 1.426 & 1.415 & 1.402 & 1.425 & 1.419 & 1.401 & 1.425 & 1.426 & 1.401 \\
$\mathrm{r}(14,15)$ & 1.226 & 1.234 & 1.234 & 1.226 & 1.232 & 1.235 & 1.226 & 1.229 & 1.235 \\
$\mathrm{r}(15,16)$ & 1.981 & 1.974 & 1.990 & 1.983 & 1.969 & 1.991 & 1.984 & 1.971 & 1.990 \\
$\mathrm{r}(16,17)$ & 2.328 & 2.317 & 2.341 & 2.329 & 2.314 & 2.341 & 2.329 & 2.319 & 2.343 \\
\hline
\end{tabular}


Table S2. Molecular orbital components of Gold diethynylfluorenyl derivatives (\%).

\begin{tabular}{|c|c|c|c|c|c|c|c|c|}
\hline \multirow{2}{*}{$\frac{\text { NO. orbital }}{98}$} & \multirow{2}{*}{$\begin{array}{l}\text { Energy/eV } \\
-0.801\end{array}$} & \multicolumn{7}{|c|}{ Composition of $\mathrm{MO}(\mathrm{Au}-\mathrm{TFT})$} \\
\hline & & & & & & $s p_{x}(\mathrm{Au})(37 \%)$ & + & $\mathrm{PH}_{3}(56 \%)$ \\
\hline 95 & -0.914 & & & $\pi^{*} p_{y}(\mathrm{C} \equiv \mathrm{C})(4 \%)$ & + & $p_{y}(\mathrm{Au})(15 \%)$ & + & $\mathrm{PH}_{3}(78 \%)$ \\
\hline 94 & -0.939 & $\pi^{*} p_{z}$ (Fluorene) (4\%) & + & $\pi^{*} p_{z}(\mathrm{C} \equiv \mathrm{C})(4 \%)$ & + & $p_{z}(\mathrm{Au})(15 \%)$ & + & $\mathrm{PH}_{3}(76 \%)$ \\
\hline 93(LUMO) & -1.247 & $\pi^{*} p_{z}$ (Fluorene) (27\%) & + & $\pi^{*} P_{\mathrm{z}}(\mathrm{C} \equiv \mathrm{C})(7 \%)$ & + & $p_{z}(\mathrm{Au})(14 \%)$ & + & $\mathrm{PH}_{3}(51 \%)$ \\
\hline 92(HOMO) & -4.790 & $\pi p_{z}$ (Fluorene) (64\%) & + & $\pi p_{z}(\mathrm{C} \equiv \mathrm{C})(28 \%)$ & + & $d_{x z}(\mathrm{Au})(4 \%)$ & + & $\mathrm{PH}_{3}(2.6 \%)$ \\
\hline 91 & -5.731 & $\pi p_{z}$ (Fluorene) (35\%) & + & $\pi p_{z}(\mathrm{C} \equiv \mathrm{C})(52 \%)$ & + & $d_{x z}(\mathrm{Au})(9 \%)$ & + & $\mathrm{PH}_{3}(3.1 \%)$ \\
\hline 90 & -6.140 & $\sigma$ (Fluorene) (15\%) & + & $\pi p_{y}(\mathrm{C} \equiv \mathrm{C})(68 \%)$ & + & $d_{x y}(\mathrm{Au})(12 \%)$ & + & $\mathrm{PH}_{3}(3 \%)$ \\
\hline NO. orbital & Energy & Composition of $\mathrm{MO}(\mathrm{Au}-\mathrm{Tl}$ & OT) & & & & & \\
\hline 101 & -0.863 & & & & & $s p_{x}(\mathrm{Au})(36 \%)$ & + & $\mathrm{PH}_{3}(57 \%)$ \\
\hline 99 & -1.011 & & & $\pi^{*} p_{y}(\mathrm{C} \equiv \mathrm{C})(3 \%)$ & + & $p_{y}(\mathrm{Au})(14 \%)$ & + & $\mathrm{PH}_{3}(79 \%)$ \\
\hline 97 & -1.242 & $\pi^{*} p_{z}$ (Fluorenone)(32\%) & + & $\pi^{*} p_{z}(\mathrm{C} \equiv \mathrm{C})(5 \%)$ & + & $p_{z}(\mathrm{Au})(12 \%)$ & + & $\mathrm{PH}_{3}(51 \%)$ \\
\hline 96(LUMO) & -1.912 & $\pi^{*} p_{z}$ (Fluorenone)(88\%) & + & $\pi^{*} p_{z}(\mathrm{C} \equiv \mathrm{C})(3 \%)$ & + & $p_{z}(\mathrm{Au})(3 \%)$ & + & $\mathrm{PH}_{3}(6 \%)$ \\
\hline 95(HOMO) & -5.102 & $\pi p_{z}$ (Fluorenone)(60\%) & + & $\pi p_{z}(\mathrm{C} \equiv \mathrm{C})(31 \%)$ & + & $d_{x z}(\mathrm{Au})(5 \%)$ & + & $\mathrm{PH}_{3}(2.7 \%)$ \\
\hline 94 & -5.966 & $\pi p_{z}$ (Fluorenone)(33\%) & + & $\pi p_{z}(\mathrm{C} \equiv \mathrm{C})(54 \%)$ & + & $d_{x z}(\mathrm{Au})(9 \%)$ & + & $\mathrm{PH}_{3}(3.1 \%)$ \\
\hline 93 & -6.219 & $\sigma$ (Fluorenone)(48\%) & + & $\pi p_{y}(\mathrm{C} \equiv \mathrm{C})(41 \%)$ & + & $d_{x y}(\mathrm{Au})(8 \%)$ & & $\mathrm{PH}_{3}(2.2 \%)$ \\
\hline 90 & -6.684 & $\pi p_{z}$ (Fluorenone)(98\%) & & & & & & \\
\hline NO. orbital & Energy & Composition of $\mathrm{MO}(\mathrm{Au}-\mathrm{Tl}$ & CNT & & & & & \\
\hline 113 & -0.897 & & & & & $s p_{x}(\mathrm{Au})(36 \%)$ & + & $\left(\mathrm{PH}_{3}\right)(57 \%)$ \\
\hline 109 & -1.485 & $\pi^{*} p_{z}(\mathrm{CN}$-Fluorene)(41\%) & + & $\pi^{*} p_{z}(\mathrm{C} \equiv \mathrm{C})(8 \%)$ & + & $p_{z}(\mathrm{Au})(12 \%)$ & + & $\left(\mathrm{PH}_{3}\right)(38 \%)$ \\
\hline 108(LUMO) & -2.774 & $\pi^{*} p_{z}(\mathrm{CN}-$ Fluorene)(98\%) & & & & & & \\
\hline 107(HOMO) & -5.265 & $\pi p_{z}(\mathrm{CN}$-Fluorene) (59\%) & + & $\pi p_{z}(\mathrm{C} \equiv \mathrm{C})(32 \%)$ & + & $d_{x z}(\mathrm{Au})(5 \%)$ & + & $\left(\mathrm{PH}_{3}\right)(2.4 \%)$ \\
\hline 106 & -6.105 & $\pi p_{z}$ (CN-Fluorene) (32\%) & + & $\pi p_{z}(\mathrm{C} \equiv \mathrm{C})(55 \%)$ & + & $d_{x z}(\mathrm{Au})(9 \%)$ & + & $\left(\mathrm{PH}_{3}\right)(2.8 \%)$ \\
\hline 105 & -6.440 & $\sigma(\mathrm{CN}-$ Fluorene) (15\%) & + & $\pi p_{y}(\mathrm{C} \equiv \mathrm{C})(68 \%)$ & + & $d_{x y}(\mathrm{Au})(13 \%)$ & + & $\left(\mathrm{PH}_{3}\right)(3.1 \%)$ \\
\hline 102 & -6.983 & $\pi p_{z}(\mathrm{CN}-$ Fluorene)(99\%) & & & & & & \\
\hline
\end{tabular}


Table S3. The Calculated Fluorescence and phosphorescence wavelengths $(\lambda)$ and their transition nature for gold diethynylfluorenyl derivatives at TD-B3LYP//TD-B3LYP level.

\begin{tabular}{lllllll}
\hline \multirow{2}{*}{ Systems } & State & \multicolumn{2}{l}{ Composition } & Nature & \multicolumn{2}{c}{$\Delta \mathrm{E}(\mathrm{eV}) / \lambda$} \\
& \multicolumn{1}{l}{$\mathrm{nm})$} & $f$ \\
\hline Au-TFT & $\mathrm{S}_{1}$ & $\mathrm{H} \leftarrow \mathrm{L}$ & $66 \%$ & $\pi \pi^{*} / \mathrm{d}_{\mathrm{xz}} \mathrm{P}$ & $2.89 / 428$ & 1.63 \\
& $\mathrm{~T}_{1}$ & $\mathrm{H} \leftarrow \mathrm{L}$ & $74 \%$ & $\pi \pi^{*}$ & $1.74 / 713$ & 0.00 \\
\hline Au-TFOT & $\mathrm{S}_{1}$ & $\mathrm{H} \leftarrow \mathrm{L}$ & $65 \%$ & $\pi \pi^{*}$ & $2.09 / 593$ & 0.22 \\
& $\mathrm{~T}_{1}$ & $\mathrm{H} \leftarrow \mathrm{L}$ & $79 \%$ & $\pi \pi^{*}$ & $1.29 / 957$ & 0.00 \\
\hline Au-TFCNT & $\mathrm{S}_{1}$ & $\mathrm{H} \leftarrow \mathrm{L}$ & $67 \%$ & $\pi \pi^{*} / \mathrm{d}_{\mathrm{xz}} \pi^{*}$ & $1.41 / 882$ & 0.05 \\
& $\mathrm{~S}_{2}$ & $\mathrm{H}-1 \leftarrow \mathrm{L}$ & $68 \%$ & $\pi \pi^{*} / \mathrm{d}_{\mathrm{xz}} \pi^{*}$ & $2.57 / 483$ & 0.01 \\
& $\mathrm{~T}_{1}$ & $\mathrm{H} \leftarrow \mathrm{L}$ & $78 \%$ & $\pi \pi^{*}$ & $0.96 / 1297$ & 0.00 \\
\hline
\end{tabular}


Table S4. Emission energies (eV) and oscillator strengths $f$ of alkynylgold(I) complexes with scalar and two-component relativistic TDDFT calculations

\begin{tabular}{|c|c|c|c|c|c|c|c|c|}
\hline \multirow{2}{*}{ Systems } & \multicolumn{3}{|c|}{ Scalar results } & \multicolumn{5}{|c|}{ Two-component results } \\
\hline & State & $\mathrm{E}(\mathrm{eV})$ & $f$ & State & $\mathrm{E}$ & $f$ & Main composition & Nature \\
\hline \multirow[t]{2}{*}{$\mathrm{Au}-\mathrm{TFT}$} & ${ }^{1} \mathrm{~A}$ & 2.777 & 1.579 & $2 \mathrm{~A}$ & 2.741 & 1.299 & ${ }^{1} \mathrm{~A}(92 \%)+{ }^{3} \mathrm{~A}(7 \%)$ & $\pi \pi^{*}$ \\
\hline & ${ }^{3} \mathrm{~A}$ & 2.081 & 0.0 & $1 \mathrm{~A}$ & 2.016 & $1.5 \times 10^{-6}$ & ${ }^{3} \mathrm{~A}(99.0 \%)$ & $\pi \pi^{*}$ \\
\hline \multirow[t]{2}{*}{$\mathrm{Au}-\mathrm{TFOT}$} & ${ }^{1} \mathrm{~A}$ & 1.695 & 0.063 & $2 \mathrm{~A}$ & 1.649 & 0.098 & ${ }^{1} \mathrm{~A}(99.7 \%)$ & $\pi \pi^{*}$ \\
\hline & ${ }^{3} \mathrm{~A}$ & 1.211 & 0.0 & $1 \mathrm{~A}$ & 1.330 & $1.4 \times 10^{-9}$ & ${ }^{3} \mathrm{~A}(99.2 \%)$ & $\pi \pi^{*}$ \\
\hline \multirow[t]{2}{*}{ Au-TFCNT } & ${ }^{1} \mathrm{~A}$ & 1.079 & 0.021 & $2 \mathrm{~A}$ & 0.906 & 0.030 & ${ }^{1} \mathrm{~A}(99.4 \%)$ & $\pi \pi^{*}$ \\
\hline & ${ }^{3} \mathrm{~A}$ & 0.805 & 0.0 & $1 \mathrm{~A}$ & 0.863 & $8.1 \times 10^{-10}$ & ${ }^{3} \mathrm{~A}(99.7 \%)$ & $\pi \pi^{*}$ \\
\hline
\end{tabular}

